Hydrology and Earth System Sciences, 3(1), 125-136 (1999) (C) EGS

\title{
UP Modelling System for large scale hydrology: deriving large-scale physically-based parameters for the Arkansas-Red River basin
}

\author{
John Ewen*, William T. Sloan, Chris G. Kilsby and P. Enda O'Connell \\ Water Resource Systems Research Laboratory, University of Newcastle, Newcastle upon Tyne, NE1 7RU, U.K. \\ * Corresponding author
}

\section{Abstract}

The UP modelling system has been applied to the $570,000 \mathrm{~km}^{2}$ Arkansas-Red River Basin (ARRB) as part of the UK NERC Terrestrial Initiative in Global Environmental Research (TIGER). The model can be run as a stand-alone basin hydrology model or be linked to existing climate and weather forecasting models. It runs on a grid comprising 1923 UP elements, each $17 \mathrm{~km}$ by $17 \mathrm{~km}$ in area, and each containing five water storage compartments: one each for the snowpack, vegetation canopy, surface water, root zone and groundwater. All the main transfers and processes of the terrestrial phase of the hydrological cycle are represented, including river network routing of the runoff from the UP elements.

The parameters of the ARRB model are physically-based, being derived either from fine-scale, sub-grid, data on the topography and physical properties of the soils, aquifers and vegetation of the basin, or from the results of fine-scale physically-based simulations. With this approach, the parameters account for the effects of sub-grid variations in moisture status and spatial distribution and are sensitive to changes in the fine-scale property data. This sensitivity is either absent or less directly represented in existing large-scale hydrology models, yet it plays a central role in studies of the impact of changes in climate and land-use.

The ARRB model, as described here and in Kilsby et al. (1999), is a first attempt at large-scale physically-based hydrological modelling of the type outlined in the 'blueprint' for the UP system (Ewen, 1997), and gives a clear, positive, indication of the nature and quality of what is currently practical with the approach.

\section{Introduction}

Based on a review of the literature, it is argued in Ewen (1997) that there is a need for a new type of large-scale basin hydrology model for application at scales up to the continental scale. This must be capable of being coupled directly to existing climate and weather forecasting models, yet must have parameters which are properly sensitive to changes in the topography and to the fine-scale physical properties of the soils, aquifers and vegetation of the basin. Such a model is needed, it is argued, as part of research into the way human actions are altering globalscale environmental properties and processes, especially those linked to changes in climate and land-use.

A 'blueprint' was given in Ewen (1997) for the UP system (the name derives from 'Upscaled Physically-based'). This was designed to meet the need for a new type of model, to be used both as a stand-alone hydrology model simulating surface and subsurface water storages and transfers, and as the hydrology component of a linked atmosphere and land surface hydrology model. The emphasis in the design of the 'blueprint' was on the physical processes known to be important in temperate climates, such as soilwater storage and flow, groundwater storage and flow, interflow, surface runoff, seasonal snowpacks, canopy storage, transpiration and evaporation. The intention was that the system would be used to study the environmental impact of pollution and changes in climate and land use, especially the impact on water resources. However, the design was not aimed at meeting the immediate needs of atmospheric modellers for improved land-surface hydrology schemes for operational climate and weather forecasting models. Rather, it was proposed as part of research contributing to future generations of operational hydrological, climate and weather models, by investigating the practicality of developing physically-based modelling approaches for large-scale hydrology which can be applied at large scales, yet account for the effects of sub-grid spatial variations in moisture status and physical properties.

The UP system has been built, and the subject of this paper and of that by Kilsby et al. (1999) is its application 
to the $570,000 \mathrm{~km}^{2}$ Arkansas-Red River Basin (ARRB), work carried out as part of the UK NERC Terrestrial Initiative in Global Environmental Research (TIGER).

In large-scale hydrological modelling, one of the central goals is usually to calibrate the simulated river flows against measured flows to ensure the model can reproduce the historical outlet hydrograph, and hence the historical behaviours for evaporation and water balance. This is not a goal in this work. As far as practical, the parameters of the UP model for the ARRB are derived from the topography and physical properties of the basin, and the central aim is to derive the parameters without calibration against river flows. The results obtained for river flows will, of course, be poorer than can be achieved by direct calibration. However, the intention with the ARRB model is to simulate the hourly water status of the basin at a full range of scales, from the point scale upwards, in a way controlled not by historical flows but by parameters derived from, and sensitive to changes in, the fine-scale properties of the basin. Such parameter sensitivity plays a central role in studies of the impact of changes in climate and land-use, yet is absent, or less directly represented, in existing largescale land-surface hydrology models.

The UP system comprises two parts. The first part is the simulation model, which is the part used to simulate the hydrology of large basins, and the second part is a set of physically-based, distributed (PBD) parametrization models, from which the parameters for the simulation model are obtained. These parametrization models have variables and parameters which apply at a fine scale (e.g. the point, $100 \mathrm{~m}, 250 \mathrm{~m}$ or $1 \mathrm{~km}$ scales) so can use property data obtained from fine scale maps of elevation, vegetation cover, soil cover, aquifer properties, etc.

The basic component of the simulation model is the UP element, and the ARRB simulation model has 1923 UP elements, one element for each of 1923 gridsquares, each of which is $17 \mathrm{~km} \times 17 \mathrm{~km}$ in area (Fig. 1). Each of the UP elements has five water storage compartments (Fig. 2): one each for the snowpack, vegetation canopy, surface water, root zone and groundwater zone. The five compartments represent hydrologically distinct zones, each playing a different role in runoff generation and each having a different timescale for response. The flows between, into and out of the compartments represent physical flows. Two further compartments, the interflow and deep unsaturated percolation compartments, were included in the blueprint'. The reasons for not including these in the ARRB model are given in the Discussion section. The distributed runoff from the basin, as given by the hourly runoff from the surface water compartments of the 1923 UP elements, is routed to the outlets of the Arkansas and Red Rivers by a river network routing scheme (see Naden et al., 1999, for a description of the routing scheme).

The physical status of the water in each UP element is represented by 10 state variables (Table 1) which are updated hourly. Five of the 10 state variables, the primary state variables, are measures of the volumes of water stored in the element's five compartments, so the updating equations for these are simply hourly mass balance equations which update the storage as a function of the difference between the total rates of input and output:

$$
\Delta S=\sum_{i=1}^{N} I_{i}-\sum_{j=1}^{M} o_{j}
$$

where $\Delta S(\mathrm{~mm})$ is the change in volume stored per unit area, and there are $\mathrm{N}$ inputs to the compartment, labelled $I_{1}$ to $I_{N}(\mathrm{~mm})$, and $\mathrm{M}$ outputs, labelled $\mathrm{O}_{1}$ to $\mathrm{O}_{\mathrm{M}}(\mathrm{mm})$. The inputs are gains, either from precipitation or from other compartments, and the outputs are losses, which

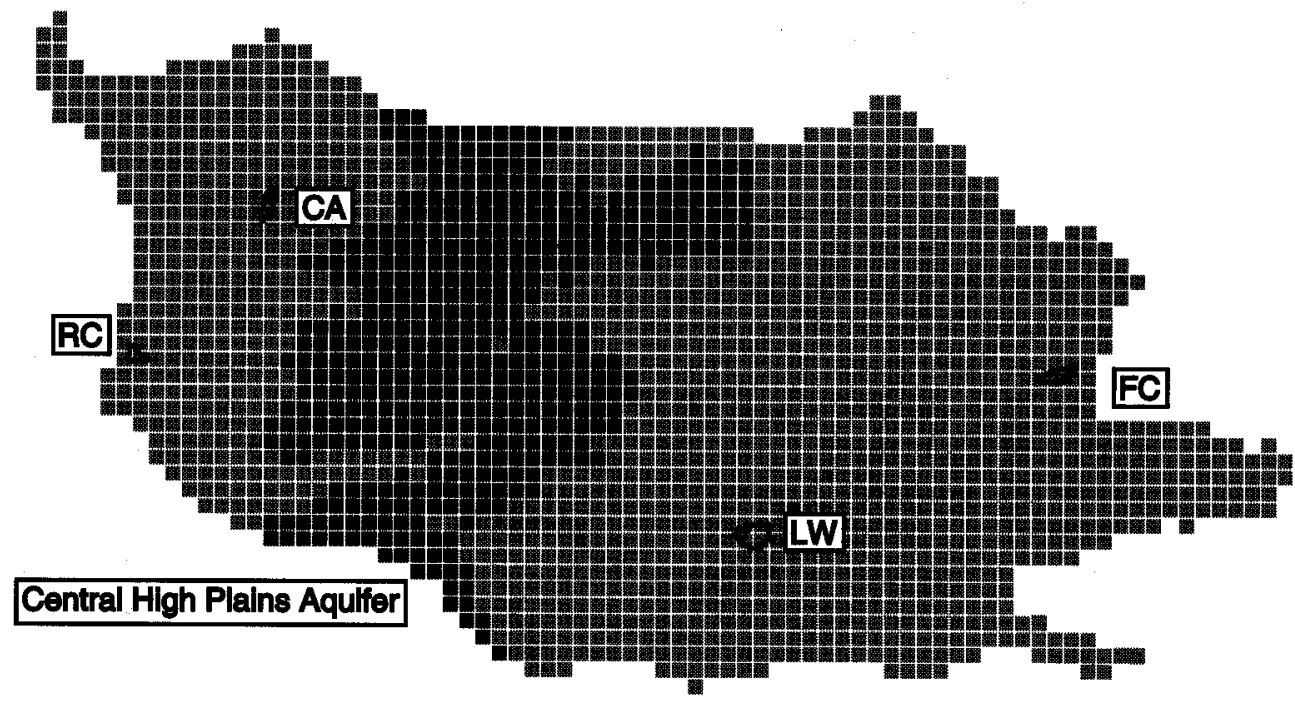

Fig. 1. The Arkansas-Red River Basin shoming the $17 \mathrm{~km} \times 17 \mathrm{~km}$ UP elements, the Central High Plains Aquifer (darker elements) and four parametrization basins: Rayado Creek (RC), Crooked Arroyo (CA), Little Washita (LW) and Flint Creek $(F C)$ 


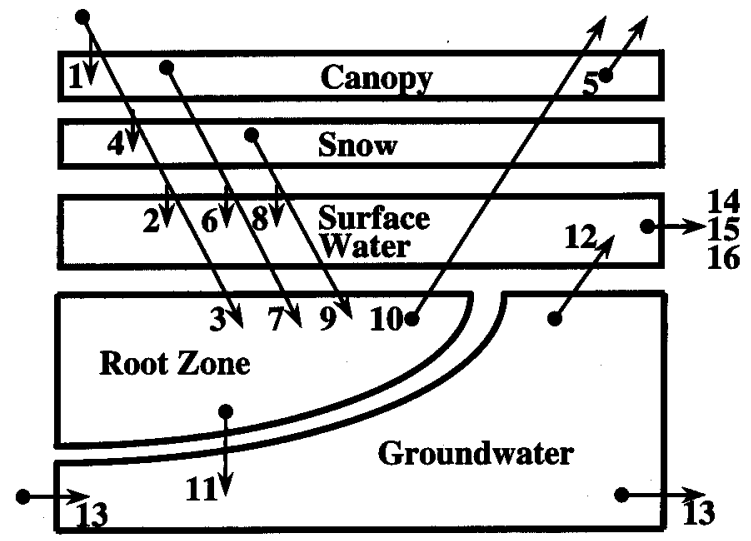

Fig. 2. The UP element (after Emen, 1997) shoming the 16 inputs and outputs corresponding to Eqns. IOI to IOI6 in Table 2 either enter other compartments (as inputs to those compartments) or are transferred to the atmosphere or to the main river network as runoff.

In total, 16 physical transfers are represented in each UP element. Each of these is simulated by water being moved either between the atmosphere and a compartment, or from one compartment to another, or, in the case of runoff, from the surface water compartment to the main river network. For each transfer, there is an equation which gives its magnitude (in $\mathrm{mm}$ ) in terms of the variables and parameters for the UP element. The equations are called the input and output equations and labelled IOI to IO16 (Table 2). The key to the UP approach is that the input and output equations are derived, as will be shown in detail in this paper, from the variables and parameters of the PBD parametrization models. The rates in the equations are for (physical) large-scale hydrological

Table 1. State Variables

\begin{tabular}{llll}
\hline Symbol & Description & Type & Equation \\
\hline$S_{c}$ & canopy storage $(\mathrm{mm})$ & primary & PSV1 \\
$S_{s}$ & snowpack storage $(\mathrm{mm})$ & primary & PSV2 \\
$S_{w}$ & surface water storage $(\mathrm{mm})$ & primary & PSV3 \\
$S_{r}$ & root zone storage $(\mathrm{mm})$ & primary & PSV4 \\
$S_{g}$ & groundwater storage $(\mathrm{mm})$ & primary & PSV5 \\
$S_{1}$ & accumulated snowfall since start of snowpack season $(\mathrm{mm})$ & secondary & SSV1 \\
$S_{2}$ & fraction of land area associated with shallow water tables & secondary & SSV2 \\
$S_{3}$ & fraction of $S_{r}$ associated with shallow water tables & secondary & SSV3 \\
$S_{4}$ & abstract measure associated with recent precipitation and & secondary & SSV4 \\
& snowmelt in regions with deep water tables $(\mathrm{mm})$ & & \\
$S_{5}$ & As $S_{4}$ but for shallow water tables $(\mathrm{mm})$ & secondary & SSV5 \\
\hline
\end{tabular}

Table 2. Input and Output Equations

\begin{tabular}{lllll}
\hline Symbol & Name & Comes From & Goes To & Equation \\
\hline$P_{c}$ & rainfall & atmosphere & canopy & IO1 \\
$P_{p}$ & rainfall & atmosphere & surface water & IO2 \\
$P_{r}$ & rainfall & atmosphere & root zone & IO3 \\
$P_{s}$ & snowfall & atmosphere & snowpack & IO4 \\
$E_{c}$ & evaporation & canopy & atmosphere & IO5 \\
$T_{w}$ & throughfall & canopy & surface water & IO6 \\
$T_{r}$ & throughfall & canopy & root zone & IO7 \\
$M_{w}$ & snowmelt & snowpack & surface water & IO8 \\
$M_{r}$ & snowmelt & snowpack & root zone & IO9 \\
$E_{r}$ & evapotranspiration & root zone & atmosphere & IO10 \\
$Q$ & percolation & root zone & groundwater & IO11 \\
$D$ & exfiltration & groundwater & surface water & IO12 \\
$G$ & exchange & groundwater & adjacent gridsquares & IO13 \\
$R^{e}$ & exfiltration runoff & surface water & main river & IO14 \\
$R^{i}$ & infiltration excess runoff & surface water & main river & IO15 \\
$R^{b}$ & saturated excess runoff & surface water & main river & IO16 \\
\hline
\end{tabular}


transfers. However, there is no 'standard' theory in the literature laying down equations which link the rates to large-scale variables, and such a theory, the theory of large-scale hydrology, is probably some years off. The process of 'parametrizing' the ARRB model therefore involves three stages: defining the (large-scale) variables, defining the input and output equations, and evaluating the (large-scale) parameters which appear in the input and output equations.

The third stage above involves evaluating the (largescale) parameters of the input and output equations using the (fine-scale) PBD models. It is, therefore, reasonable to describe the input and output equations as large-scale physically-based equations and their parameters as largescale physically-based parameters. The method of deriving some of the parameters implicitly involves 'upscaling' in that spatially distributed fine-scale physical property data and/or simulation output from the PBD models are aggregated to the scale of the UP element before being used in the parametrization. Some of the parameters are calibrated against aggregated data, but the ARRB model is not 'calibrated' in the traditional sense of a basin model calibrated against measured river flows.

The input and output equations are algebraic equations. However, look-up tables are used in the snowpack equations, and these tables are read using linear interpolation. The full parameter set for the ARRB model, therefore, comprises the parameters of the algebraic equations and the table values in the look-up tables.

There are two types of variables in the ARRB model: forcing variables (Table 3 ), which are prescribed or calculated hourly for each gridsquare; and state variables (Table 1). The five secondary state variables in Table 1 are updated hourly using Eqns. SSV1 to SSV5, which, in effect, define the secondary state variables as functions of the forcing variables and primary state variables. The secondary state variables are used as measures of the sub-grid moisture status and/or the spatial distribution of the water in the compartments, and their role is, explicitly, to include this information in the input and output equations.

For the current version of the ARRB model, there is no sub-grid routing of surface water, so there is no storage of surface water (i.e. $S_{w}=0$ ), and water arriving in the sur- face water compartment is simply transferred immediately to the main river network.

The full set of equations for the ARRB model has now been introduced. In summary, this comprises:

- the input and output equations, IO1 to IO16

- the updating equations for the primary state variables, PSV1 to PSV5 (each of which has the form of Eqn. 1)

- and the updating equations for the secondary state variables, SSV1 to SSV5.

Owing to lack of space, only a subset of these equations is presented here.

\section{Deriving large-scale parameters}

The parameters in the input and output equations are listed in Table 4, and the parametrization models used to obtain the parameters are listed in Table 5 . The parametrization models were initially applied to only three representative basins: Little Washita (LW; $600 \mathrm{~km}^{2}$ ); Crooked Arroyo (CA; $275 \mathrm{~km}^{2}$ ); and Rayado Creek (RC; $165 \mathrm{~km}^{2}$ ); (see Fig. 1 and Kilsby et al., 1999). These three each have areas similar to the area of a gridsquare $(17 \mathrm{~km} \times 17 \mathrm{~km}=$ $289 \mathrm{~km}^{2}$ ), so parameters derived for the basins were assumed to apply directly to gridsquares, without adjustment or scaling. How the parameters were used throughout the area of the ARRB is described in Kilsby et al. (1999).

As a refinement on this initial parametrization, two further parameter sets were derived, for the areas around McAlester (McA) and Flint Creek (FC; $296 \mathrm{~km}^{2}$ ). McAlester lies only $220 \mathrm{~km}$ east of Little Washita but, for the parametrization period (June 1984 - May 1986, inclusive), it had $1233 \mathrm{~mm}$ precipitation compared to $804 \mathrm{~mm}$ at Little Washita. The McAlester parameters were derived using the property data for Little Washita but the precipitation and meteorological data for McAlester. This was done to improve the quality of the simulated hydrological response for the area to the east of Little Washita, and as a simple study of the sensitivity of the ARRB model parameters to precipitation forcing.

The hydrological behaviour of the area around Flint Creek is poorly understood: it is karst, and there are few

Table 3. Forcing Variables

\begin{tabular}{lll}
\hline Symbol & Description & Sub-Grid Distribution \\
\hline$p$ & precipitation & uniform \\
$T^{\prime}$ & air temperature at reference point & point \\
$v$ & vapour pressure deficit & uniform \\
$i$ & net irradiance & uniform \\
$f_{c}$ & fractions of land area covered by bare & uniform \\
& soil and each of three types of vegetation & \\
\hline
\end{tabular}


Table 4. Large-Scale Parameters

\begin{tabular}{|c|c|c|c|c|c|c|}
\hline Symbol & Description & Units & $\begin{array}{l}\text { No. per } \\
\text { Gridsquare }\end{array}$ & $\begin{array}{l}\text { Derivation } \\
\text { Method }\end{array}$ & Used in & Source \\
\hline C & canopy capacity (time varying) & $\mathrm{mm}$ & 3 & adopted & IO1, 5-7,10 & M5 \\
\hline$c$ & storage coefficient for inter-grid exchange & - & 1 & See main text & IO13 & M9 \\
\hline$L$ & lapse rate & $\mathrm{K} \mathrm{m}^{-1}$ & 1 & adopted & IO4,8-9 & M2 \\
\hline$r_{a}$ & aerodynamic resistance (time varying) & $\mathrm{hr} \mathrm{mm}^{-1}$ & 4 & adopted & 105,10 & M6 \\
\hline$r_{c}$ & canopy resistance (time varying) & hr $\mathbf{m m}^{-1}$ & 4 & adopted & 105,10 & M6 \\
\hline$T_{x}, T_{y}$ & transmissivities for inter-grid exchange & $\mathrm{mm}^{2} \mathrm{~h}^{-1}$ & 1 of each & See main text & IO13 & M9 \\
\hline$T_{f}$ & snowfall threshold & ${ }^{\circ} \mathrm{C}$ & 1 & adopted & IO4 & M3 \\
\hline$T_{0}$ & melt threshold & ${ }^{\circ} \mathrm{C}$ & 1 & adopted & IO8-9 & M4 \\
\hline$z^{\prime}$ & reference elevation & $\mathrm{m}$ & 1 & adopted & $\mathrm{IO4,8-9}$ & M1 \\
\hline$\Lambda$ & fraction of area lying above given elevation & - & look-up table & aggregated & $\mathrm{IO} 4,8-9$ & M1 \\
\hline$\alpha$ & evapotranspiration factors & See Table 6 & 8 & $\begin{array}{l}\text { aggregated/ } \\
\text { calibrated }\end{array}$ & IO5,10, SSV4-5 & M7 \\
\hline$\beta$ & percolation factors & See Table 6 & 8 & $\begin{array}{l}\text { aggregated/ } \\
\text { calibrated }\end{array}$ & IO11 & M7 \\
\hline$\gamma$ & infiltration excess factors & See Table 6 & 4 & $\begin{array}{l}\text { aggregated/ } \\
\text { calibrated }\end{array}$ & $\mathrm{IO} 2,15$ & M7 \\
\hline$\delta$ & exfiltration factors & See Table 6 & 4 & $\begin{array}{l}\text { aggregated/ } \\
\text { calibrated }\end{array}$ & $\mathrm{IO} 12,14$ & M8 \\
\hline$\varepsilon$ & saturated area factor & $\mathrm{mm}^{-1}$ & 1 & $\begin{array}{l}\text { aggregated/ } \\
\text { calibrated }\end{array}$ & $\mathrm{IO} 2,16$ & M8 \\
\hline$\xi$ & frozen fraction of land surface & - & 1 & adopted & 108,15 & M7 \\
\hline$\eta$ & Interim, for FC only & - & 6 & See main text & See main text & M11 \\
\hline
\end{tabular}

Table 5. Parametrization Models. Use number: 1, aggregation calculations for evaluating parameters and variables; 2 , basis for 'upscaled' equation; 3, adopted at large-scale, without scaling or other adjustment; 4, fine-scale or patch simulations to obtain data for calibrating large-scale parameters; 5 , grid-scale simulations to obtain large-scale parameters; 6 , interim model, to be replaced at later date. The basins are: Crooked Arroyo (CA); Flint Creek (FC); Little Washita (LW); McAlester (McA); Rayado Creek (RC)

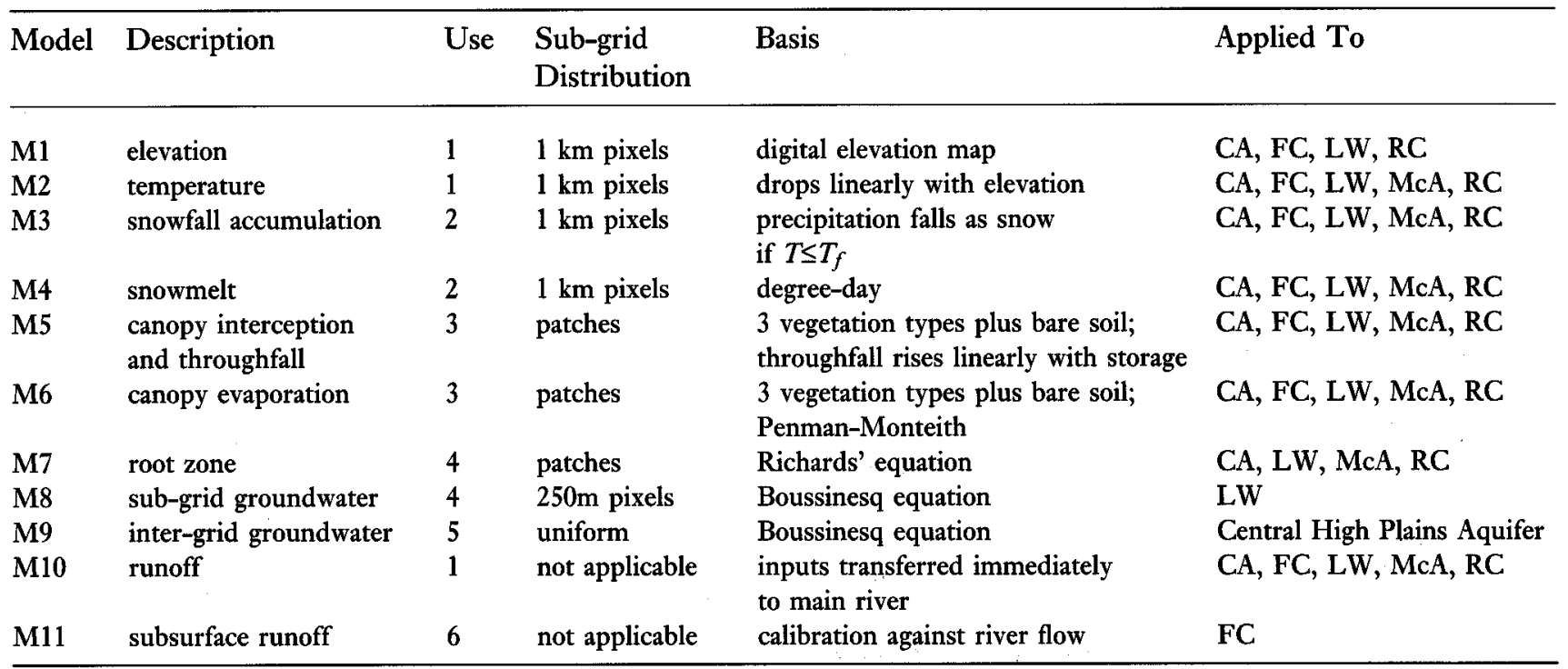


reliable data available for the hydraulic properties in the region. As an interim measure, therefore, the root zone and groundwater compartment equations for this region were derived from measured river flows.

Some of the parametrization models are very simple; for example, model $\mathrm{M} 2$ can be written as:

$$
T(z)=T\left(z^{\prime}\right)-L\left(z-z^{\prime}\right)
$$

which gives the temperature at elevation $\mathrm{z}$ within the gridsquare in terms of the temperature at the reference elevation, $z^{\prime}$, and the lapse rate, $L$. The most complicated parametrization model is $M 7$, which is a fully coupled one-dimensional soil-vegetation-atmosphere finite-difference scheme for simulating moisture transfers in plots comprising a stack of horizontal layers of different soil types, containing a water table and covered by any combination of three types of vegetation and bare soil (treated in a way consistent with M5 and M6). M7 also includes a snowpack (treated in a way consistent with M3 and M4) and a mechanism for infiltration excess runoff.

In Table 4, in the column entitled 'derivation method', 'Adopted' indicates that the parametrization model is assumed to apply at both the fine scale and the large scale, and the fine scale parameters are adopted (i.e. used directly, without scaling or other processing) at the large scale. For example, the canopy capacity for interception water storage in oak forest is assumed to hold equally at the fine and large scales (note, at the large scale there can be a mix of bare soil and up to three vegetation types, each covering a specified fraction of the ground surface, so the large-scale canopy capacity parameter does not require 'upscaling' to account for incomplete ground coverage).

'Aggregated' in Table 4 indicates that the derivation of the large-scale parameters involves simple integration of small-scale data over the area of the parametrization basin. For example, the look-up table values for $\Lambda$ are calculated, by integration, directly from the digital elevation map (i.e. model M1), and the exfiltration data used in the calibration of the exfiltration parameters, $\delta$, are calculated by integration from $250 \mathrm{~m}$ exfiltration maps produced by running model M8.

'Calibrated' in Table 4 indicates that the large-scale parameters are evaluated by calibration against simulation results produced by running the parametrization model. For example, the $\delta$ parameters in Eqns. IO12 and IO14 were calibrated against hourly time-series for the total exfiltration rate and total groundwater storage, both series being calculated by aggregation of $250 \mathrm{~m}$ hourly results from model M8.

'Pixels' and 'patches' in Table 5 refer to the treatment of the fine-scale variables and parameters in the parametrization models. 'Patches' indicates that the parametrization basin is treated as a collection of independent uniform patches. Each patch simulated by the root zone parametrization model, M7, for example, represents a different combination of soils, vegetation cover and depth to the water table, and the aggregation process for the results from the patch simulation involves simple summation which takes into account the land area associated with each patch.

The derivation of some of the large-scale parameters is described in detail in the following sections.

\section{SNOWPACK}

The snowpack model was developed from first principles for use in the UP system, and involves the direct integration of the fine-scale equation for snowmelt (this is the only case where a fine-scale equation is thus integrated). The fine-scale parametrization models are M2, given earlier, plus:

$$
\Delta s= \begin{cases}p & \text { if } T(z) \leq T_{f} \\ 0 & \text { otherwise }\end{cases}
$$

and

$$
m= \begin{cases}k\left(T-T_{0}\right) & \text { if } T>T_{0} \\ 0 & \text { otherwise }\end{cases}
$$

where $k$ is the degree day factor, $p$ precipitation rate, $T_{f}$ air temperature at and below which precipitation falls as snow, $T_{0}$ melt threshold temperature, $m$ melt rate, and $\Delta \mathrm{s}$ the change in the depth of water in the snowpack. (It is recognised that a stronger claim to physical basis could be made if an energy budget approach were used in place of the degree-day approach.) The snow compartment equations are (see Tables $1-4$ for nomenclature):

$$
\begin{aligned}
& \Delta S_{s}=P_{s}-M_{w}-M_{r} \\
& \Delta S_{1}=\left\{\begin{array}{cl}
-S_{1} & \text { at start of snowpack season } \\
\mathrm{P}_{\mathrm{s}} & \text { otherwise }
\end{array}\right. \\
& P_{s}=\Lambda\left[z^{\prime}+\left(T^{\prime}-T_{f}\right) / L\right] p \\
& M_{m}=A M \\
& M_{r}=(1-A) M
\end{aligned}
$$

where $A$, the partition coefficient, is the maximum of the fraction of land area saturated by exfiltrating groundwater (discussed later) and the fraction frozen, $\xi$ (which is taken as 1 for the Rocky Mountains and 0 elsewhere), and $M$, the total melt rate, is found from the integral of the finescale degree-day equation ( $M=\int_{m d a}$, where $a$ is area):

$M= \begin{cases}k[\Lambda(X)-\Lambda(Y)]\left(T^{\prime}+L z^{\prime}-T_{0}\right)+k L \int_{\Lambda(X)}^{\Lambda(Y)} z d \Lambda & \text { if } X \leq Y \\ 0 & \text { otherwise }\end{cases}$ 
where $Y\left[=z^{\prime}+\left(T^{\prime}-T_{0}\right) / L\right]$ is the elevation above which there is no melt, and $X$ the elevation below which there is no snowpack, i.e. the elevation of the melt front. This melt equation can be seen to have the form of a modified degree day equation, containing a scaling factor $\Lambda(X)-\Lambda(Y)$, which is the fraction of the gridsquare which contains a melting pack, and a correction term (the integral term), which is always negative and accounts for the effect of the local melt rate falling with elevation.

$\mathrm{Up}$ to this point in the development of the snowpack model, the derivation has been entirely consistent with the fine-scale models. A consistency problem arises, however, in the calculation of the elevation of the melt front, $X$. At any point on the land surface, the local snowpack depth depends on the history of local accumulation and melt, so there is no simple relationship between the time at which the melt front reaches a given elevation and the pack depth, $S_{s}$. A simple approximate relationship is therefore used in the ARRB model, in which the ratio $S_{s} / S_{1}$ is treated as a measure of the sub-grid distribution of snow, such that $X$ $=\Lambda^{-1}\left(S_{s} / S_{1}\right)$. This, for example, assumes that if half the seasonal snowpack has melted, the pack has completely melted over half the gridsquare. Alternative, improved, formulations for describing the sub-grid distribution are being sought. The ARRB snowpack model includes a sub-model, not described anywhere in this paper, which allows for the relatively minor effects associated with the re-freezing and through-pack routing of melt water.

\section{ROOT ZONE}

The equation at the heart of the parametrization model M7, for the root zone, is Richards' equation:

$$
\frac{\partial \theta}{\partial t}=\frac{\partial}{\partial z}\left(K \frac{\partial \psi}{\partial z}+K\right)-\sigma P E
$$

where $t$ is time, $z$ elevation, $\theta$ is the volumetric moisture content, $K(\theta)$ the unsaturated hydraulic conductivity function, $\psi(\theta)$ the matric potential function, and PE the potential evaporation rate calculated using M6. The factor $\sigma$ represents the variation over depth of the water uptake, accounting for the effect of the reduction of transpiration associated with evaporation from the canopy storage (reduced by a fraction equal to the ratio of canopy storage to canopy capacity), and the reduction associated with water stress. The ground cover can have up to three vegetation types and bare soil. For each of the three types, the fine-scale parameters for transpiration are tables of root fraction over depth, and the upper and lower matric potentials between which the transpiration reduction fraction for water stress varies linearly between 0 and 1 .

M7 was applied over the full depth of the root zone. At the ground surface, there is infiltration, bare soil evaporation, and runoff during ponding. At the base of the root zone, the matric potential is fixed at a value corresponding to the vertical distance between the base and the water table (this, approximate, approach was adopted since good data were not available for the percolation properties of the deeper sections of the unsaturated zone).

The most complete implementation of $\mathrm{M} 7$ was for the Little Washita basin, which is the only basin with a local groundwater aquifer substantial enough to require the use of the sub-grid groundwater model, M8. Eight, 2-year, hourly patch simulations were run, one each for a shallow and a deep water table for each of four patches. Preliminary simulations were run to obtain self-consistent initial moisture profiles. The patches were chosen to represent the four main soil and vegetation combinations seen in the Little Washita, and the areas the patches cover and their property data were obtained from maps of vegetation, soils, etc. (see Kilsby et al., 1999). The shallow water table was assumed fixed at $3 \mathrm{~m}$ below ground, a representative value based on the results from M8. (To ensure overall consistency of results, M8 used the M7 simulated percolation as recharge.) The patches for the shallow water table represent areas near streams, where the subsurface response is relatively rapid and linked to rapid changes in exfiltration. The depth to the deep water table, based on borehole data and results from M8, varies from patch to patch, but is at least $12 \mathrm{~m}$. In total, $87.14 \%\left(=1-S_{2}\right)$ of the Little Washita land area is associated with deep water tables. The percolation water from this area supplies the bulk of the exfiltration water, resulting in the exfiltration having a relatively smooth yearly cycle as a consequence of the percolation waters from the different parts of the basin having different sub-surface lateral-flow travel times.

The updating equation for the primary state variable for the root zone is:

$$
\Delta S_{r}=P_{r}+T_{r}+M_{r}-E_{r}-Q
$$

The $P_{r}, T_{r}$ and $M_{r}$ terms are inputs to the root zone, controlled by the forcing variables and the outputs from the canopy and snowpack compartments, and the $E_{r}$ and $Q$ terms are outputs, given by the sum of rates for deep (superscript $d$ ) and shallow (superscript $s$ ) water tables:

$$
\begin{aligned}
& E_{r}=\left(1-S_{2}\right) E_{r}^{d}+S_{2} E_{r}^{s} \\
& Q=\left(1-S_{2}\right) Q^{d}+S_{2} Q^{s}
\end{aligned}
$$

The parameters for these are given in the following equations, which were created and calibrated by testing against the aggregated $M 7$ plot simulation data:

$$
\begin{aligned}
E_{r}^{d} & =\min \left(1, \max \left\{0, \alpha_{1} S_{4}+\alpha_{2} S^{d}-\alpha_{3}\right\}\right) P E \\
E_{r}^{s} & =\min \left(1, \max \left\{0, \alpha_{5} S_{5}+\alpha_{6} S^{s}-\alpha_{7}\right\}\right) P E \\
Q^{d} & =\max \left\{0, \beta_{1}\left(S^{d}-\beta_{2}\right)\right\}+\max \left\{0, \beta_{3}\left(S^{d}-\beta_{4}\right)\right\} \\
Q^{s} & =\max \left\{0, \beta_{5}\left(S^{s}-\beta_{6}\right)\right\}+\max \left\{0, \beta_{7}\left(S^{s}-\beta_{8}\right)\right\}
\end{aligned}
$$

where $S^{d}$ and $S^{s}$ are, respectively, the storages associated with deep and shallow water table areas, as given by the 
following functions of state variables:

$$
S^{d}=\left(1-S_{3}\right) S_{r} /\left(1-S_{2}\right)
$$

and

$$
S^{s}=S_{3} S_{r} / S_{2}
$$

The secondary state variable $S_{2}$ is defined above, and the updating equations for secondary state variables, $S_{3}, S_{4}$ and $S_{5}$, which appear in the above equations are:

$$
\begin{aligned}
& \Delta S_{3}=\left(P_{r}+T_{r}+M_{r}-E_{r}^{s}-Q^{s}-S_{3} \Delta S_{r}\right) / S_{r} \\
& \Delta S_{4}= \begin{cases}F-\alpha_{4} P E & \text { if }\left(F-\alpha_{4} P E\right)>-S_{4} \\
-S_{4} & \text { otherwise }\end{cases}
\end{aligned}
$$

and

$$
\Delta S_{5}= \begin{cases}F-\alpha_{8} P E & \text { if }\left(F-\alpha_{8} P E\right)>-S_{5} \\ -S_{5} & \text { otherwise }\end{cases}
$$

where $F$, the rate water reaches the ground surface, is given by:

$$
F=P_{r}+T_{r}+M_{r}
$$

The role of the secondary state variables $S_{4}$ and $S_{5}$ is to increase bare soil evaporation and transpiration when the root zone contains new water which arrived recently in the zone. They are, in effect, measures of the vertical distribution of moisture in the soil profile; the higher their values the wetter are the upper soil layers, but they do not represent real water so are abstract measures.

Following the same approach as above, aggregated patch time-series were also used to create and calibrate an equation for infiltration excess runoff:

$$
\begin{aligned}
R^{i} & =\left(1-S_{2}\right) R^{i d}+S_{2} R^{i s} \\
& =\left(1-S_{2}\right) \gamma_{1} \max \left\{0, F-\gamma_{2}\right\}+S_{2} \gamma_{3} \max \left\{0, F-\gamma_{4}\right\}
\end{aligned}
$$

All the calibrated parameters of the above equations are positive (Table 6). The calibration algorithm uses a multidimensional minimisation downhill simplex method with annealing (Press et al., 1992). The four parameters $\alpha_{1}$ to $\alpha_{4}$, for example, were found simultaneously by minimising the 'difference' between the aggregated M7 plot evapotranspiration rate for deep water table areas and the rate produced by running the $E_{r}^{d}$ and SSV4 equations with aggregated M7 data for $S^{d}$ and PE. The 'difference' which is minimised is the sum of the root mean square hourly difference and the cumulative difference for the full period of the simulation. A combined 'difference' of this kind was used in all the calibrations, to ensure the calibrated equations capture the short term fluctuations in response and give good estimates of the long-term mass balance.

Figure 3 shows the forcing variables for Little Washita. Figures 4 to 8 are for the deep water table areas at Little Washita. These give an indication of the quality of fit achieved in the calibrations, and Fig. 6, especially, gives some insight into the parametrization process. Fig. 6 shows there is hysteresis in the relationship between percolation and root zone storage (which is associated both with the infiltration-to-percolation travel time being different between patches and with the inherent nonlinearity of moisture flow in unsaturated soil; see Ewen, 1997, for discussions on hysteresis). In creating the percolation equation, IO11, however, the hysteresis has been neglected, and a piece-wise linear relationship assumed to hold between percolation and storage. The result, as can be seen in Fig. 7, is that Eq. IO11 does not reproduce the aggregated M7 patch percolation data exactly, but does capture the main features of percolation and gives very good long-term estimates of the cumulative percolation. To improve Eqn. IO11, secondary state variables, similar to $\mathrm{S}_{4}$ or to one of the other types described in Ewen (1997), would need to be introduced.

\section{GROUNDWATER}

Parametrization model M8 is the Boussinesq equation for phreatic aquifers:

$$
c \frac{\partial h}{\partial t}=\frac{\partial}{\partial x}\left(T_{x} \frac{\partial h}{\partial x}\right)+\frac{\partial}{\partial y}\left(T_{y} \frac{\partial h}{\partial y}\right)+r .
$$

where $c$ is the storage coefficient, $h$ the elevation of the phreatic surface, $T_{x}$ and $T_{y}$ are, respectively, the transmissivities in the $x$ and $y$ directions, and $r$ the recharge rate. M8 was applied to Little Washita on a $250 \mathrm{~m} \times$ $250 \mathrm{~m}$ finite-difference grid, using the percolation from M7 as recharge. Initial elevations for the phreatic surface were first established, then a two-year, daily simulation run.

The sole updating equation for the groundwater zone is:

$$
\Delta S_{g}=Q-D
$$

and the following equation was created and calibrated using the aggregated M8 data for exfiltration rate $D$ and storage $S_{g}$ :

$$
D=\delta_{1} \sin \left(2 \pi t-\delta_{2}\right)+\delta_{3} S^{s}\left(S_{g}+\delta_{4}\right)
$$

This has an annual sinusoidal term and a more dynamic term for the rapid response to rainfall and melt (Fig. 9). The dynamic term is controlled by $S^{s}$, given previously as a function of the state variables for the root zone compartment, which is treated as a secondary state variable for the groundwater compartment. The greater is $S^{s}$, the wetter the near-stream area of the root zone, and the greater the groundwater exfiltration rate. (Note, since exfiltrating water is transferred immediately to the main river network, Eqn. IO14 can be written as: $R^{e}=D$.)

The rate of saturation excess runoff is taken as the product of the rate that water reaches the ground surface, $F$, 
Table 6. Examples of Large-Scale Parameters Calibrated Using Output from PBD Modelling

\begin{tabular}{llllll}
\hline Parameter & Units & Little Washita & McAlester & Crooked Arroyo & Rayado Creek \\
\hline$\alpha_{1}$ & $\mathrm{~mm}^{-1}$ & 0.0183855 & 0.0148128 & 0.0350294 & 0.0111940 \\
$\alpha_{2}$ & $\mathrm{~mm}^{-1}$ & 0.00604152 & 0.00740694 & 0.00557493 & 0.00328599 \\
$\alpha_{3}$ & - & 0.822930 & 1.05203 & 0.623553 & 0.496849 \\
$\alpha_{4}$ & - & 2.21077 & 2.72870 & 1.55157 & 0.752174 \\
$\alpha_{5}$ & $\mathrm{~mm}^{-1}$ & 0.0287814 & 0.00104414 & 0.0122935 & 0.0 \\
$\alpha_{6}$ & $\mathrm{~mm}^{-1}$ & 0.00726340 & 0.00622012 & 0.00843527 & 0.0 \\
$\alpha_{7}$ & - & 1.05799 & 0.817422 & 1.31942 & 0.0 \\
$\alpha_{8}$ & - & 2.20838 & 1.25741 & 1.51267 & 0.0 \\
$\beta_{1}$ & - & 0.00384830 & 0.00429064 & $5.77651 \times 10^{-5}$ & $1.41850 \times 10^{-6}$ \\
$\beta_{2}$ & $\mathrm{~mm}$ & 291.961 & 303.414 & 138.702 & 694.975 \\
$\beta_{3}$ & - & 0.000356902 & 0.000723733 & 0.0 & 0.0 \\
$\beta_{4}$ & $\mathrm{~mm}$ & 195.050 & 218.168 & 0.0 & 0.0 \\
$\beta_{5}$ & - & 0.00833438 & 0.00774804 & 0.00047082 & 0.0 \\
$\beta_{6}$ & $\mathrm{~mm}$ & 303.266 & 289.710 & 226.891 & 0.0 \\
$\beta_{7}$ & - & 0.00250991 & 0.00153271 & 0.0 & 0.0 \\
$\beta_{8}$ & $\mathrm{~mm}$ & 256.178 & 237.165 & 0.0 & 0.0 \\
$\gamma_{1}$ & - & 0.384195 & 0.400876 & 0.0132782 & 0.0317135 \\
$\gamma_{2}$ & $\mathrm{~mm}$ & 17.0043 & 15.0614 & 7.13507 & 1.491586 \\
$\gamma_{3}$ & - & 0.408995 & 0.521212 & 0.0134664 & 0.0 \\
$\gamma_{4}$ & $\mathrm{~mm}$ & 11.0349 & 13.9077 & 7.03852 & 0.0 \\
$\delta_{1}$ & $\mathrm{~mm}$ & 0.00218821 & - & - & - \\
$\delta_{2}$ & $\mathrm{rad}$ & $1.498 \pi$ & - & - & - \\
$\delta_{3}$ & $\mathrm{~mm}$ & $1.42001 \times 10^{-7}$ & - & - & - \\
$\delta_{4}$ & $\mathrm{~mm}$ & 244.147 & - & - & - \\
$\varepsilon$ & $\mathrm{mm}$ & 0.000151234 & - & - & - \\
\hline & & & & & \\
\hline
\end{tabular}

and the fraction of the ground surface which is saturated by groundwater exfiltration:

$$
R^{b}=F \epsilon S^{s}
$$

where $\varepsilon$ was calibrated against the aggregated M8 data for exfiltration saturated area.

\section{GROUNDWATER EXCHANGE}

Model M9, for groundwater exchange between the groundwater compartments in adjacent gridsquares, is based on the same equation as M8, but applied at the grid scale (i.e. on a $17 \mathrm{~km} \times 17 \mathrm{~km}$ finite-difference grid). M9 was used for the Central High Plains Aquifer $(125,000$ $\mathrm{km}^{2}$ ), which is the only major regional aquifer in the ARRB. Data for M9 were drawn from maps of long-term average recharge and borehole values of saturated depths and conductivities, and a map of transmissivity (Fig. 10) derived from steady simulation results from M9. The derived transmissivities are the large-scale parameters for groundwater exchange and are used in the groundwater exchange equation, IO13, which is simply a finitedifference analogue equation for the Boussinesq equation, applied at the $17 \mathrm{~km} \times 17 \mathrm{~km}$ scale with percolation, $Q$, as recharge and exfiltration, $D$, calculated as the discharge rate upwards through the ground surface.

\section{Discussion and Conclusions}

A major effort went into designing and building the ARRB model. It is original, detailed and comprehensive, but there are several areas which could be improved if further data were obtained and further development work undertaken. With further data, the interim modelling for Flint Creek could be replaced, and with data for further parametrization basins, the overall parametrization of the ARRB model could be improved. UP compartments for interflow and unsaturated percolation could be added to bring the ARRB model up to the full specification in the 'blueprint' given in Ewen (1997). These were not included in the current model owing to lack of data on the physical properties for near-surface sub-horizontal flow and deep vertical flow. However, the gain in adding these may not be substantial, since the root zone and groundwater compartments already account for the most important fast and slow processes for vertical and lateral subsurface flow; at 

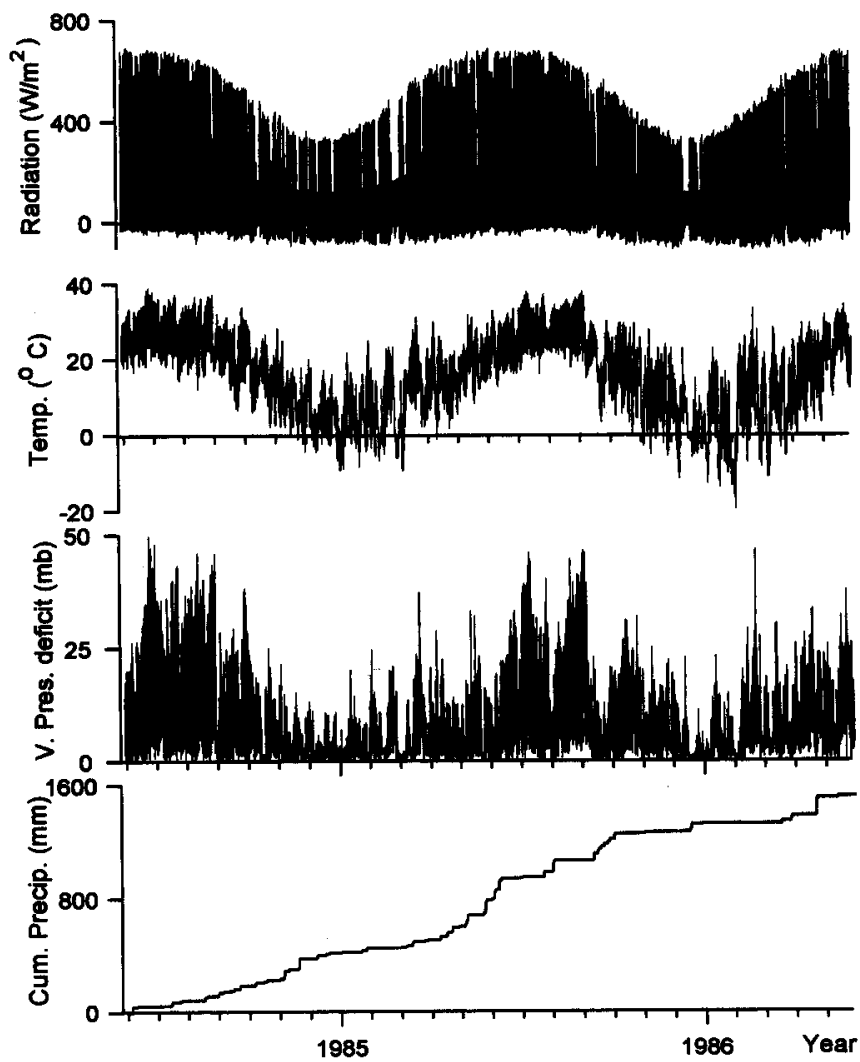

Fig. 3. Forcing variables for Little Washita

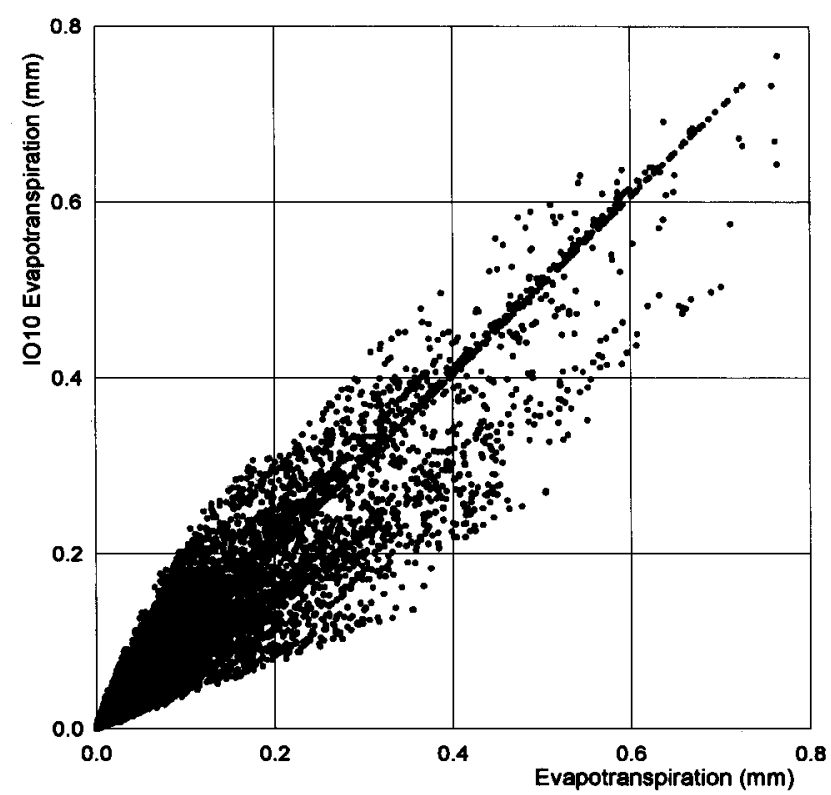

Fig. 4. Evapotranspiration by Eqn. IOIO for deep mater table regions in Little Washita plotted against the aggregated $M 7$ patch simulation values against which IO10 mas calibrated (for a perfect calibration the points would lie on a line with unit slope)

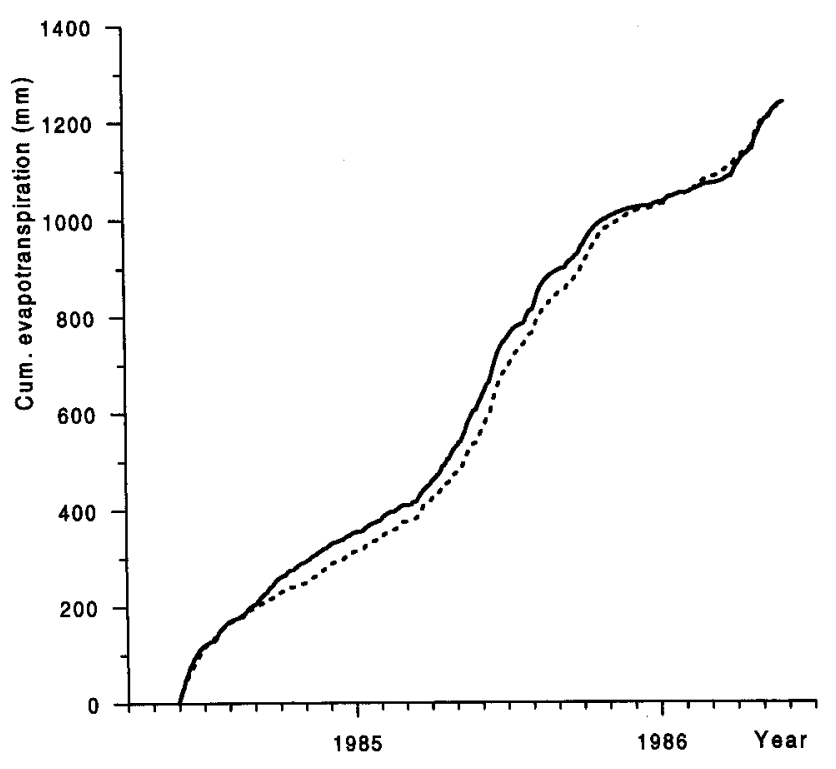

Fig. 5. Cumulative evapotranspiration for deep water table regions in Little Washita. For Eqn. IO1O (- - - -) and the aggregated $M 7$ patch simulations (-

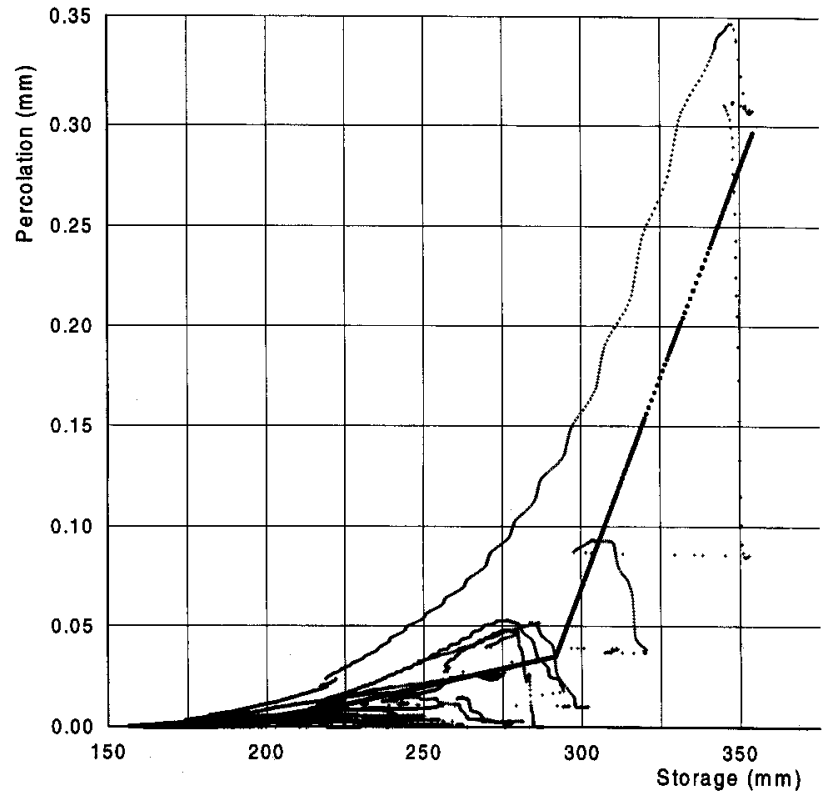

Fig. 6. Percolation versus root zone storage for deep water table regions in Little Washita. For Eq. IOII (......) and the aggregated M7 patch simulations (........) 

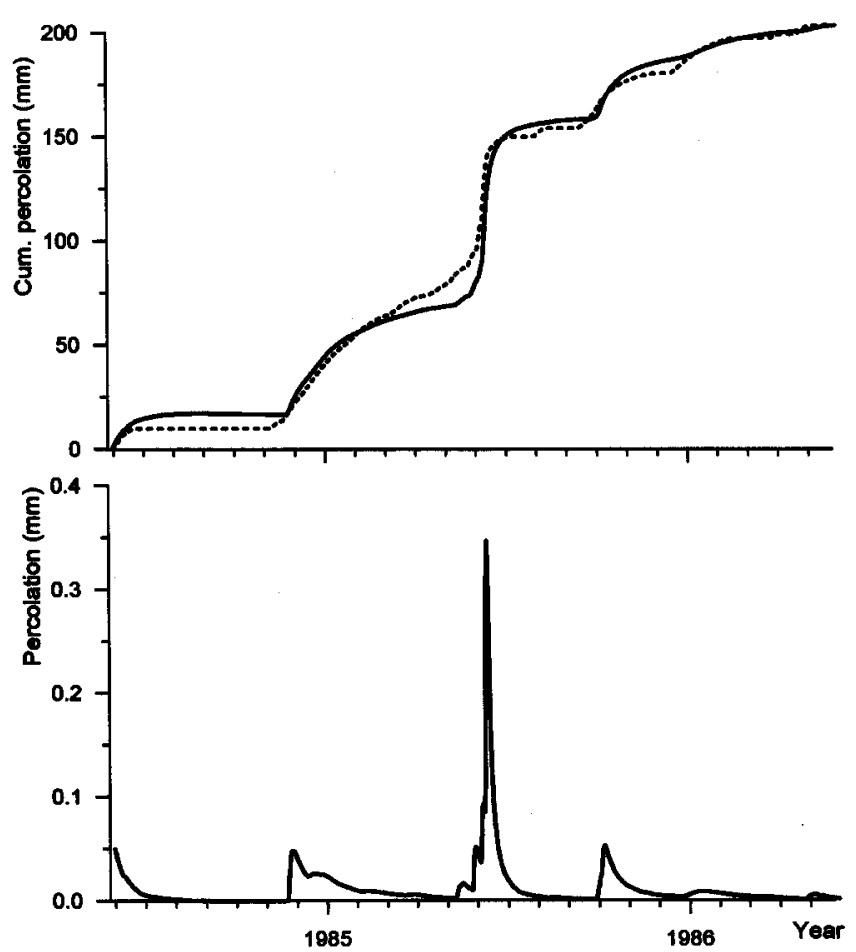

Fig. 7. Percolation and cumulative percolation for deep water table regions in Little Washita. For IOII (cumulative plot only; - - - -) and the aggregated $M 7$ patch simulations (-

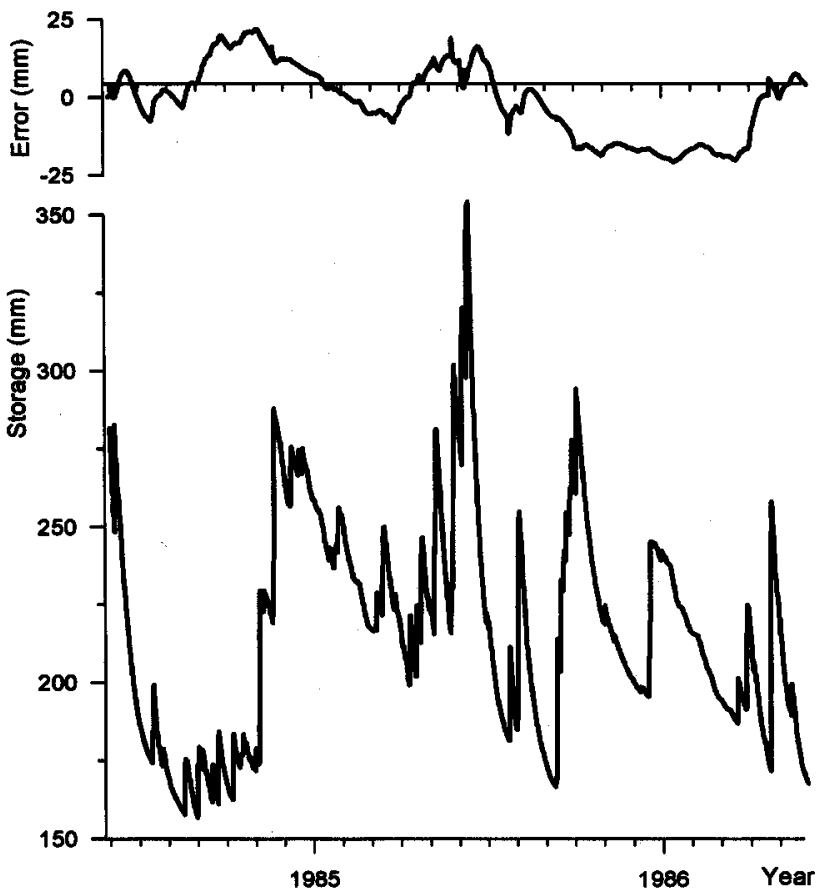

Fig. 8. Root zone storage for the aggregated $M 7$ patch simulations for deep mater table regions in Little Washita, and the error in using Eqn. PSV4 to simulate the storage

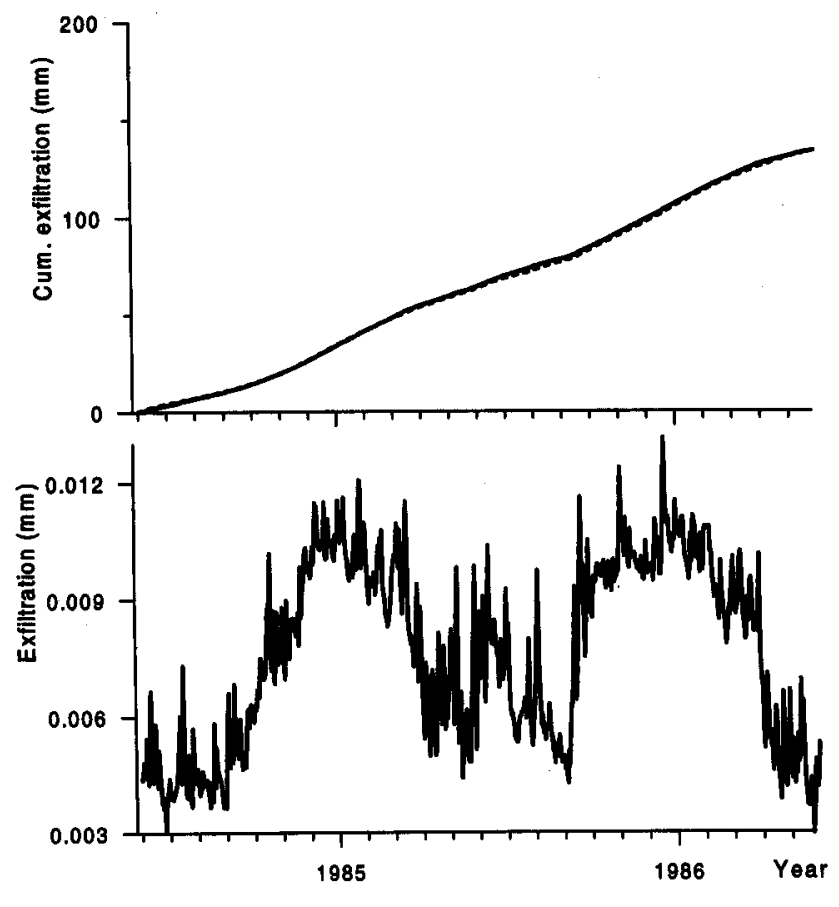

Fig. 9. Exfltration and cumulative exflitration for Little Washita. For Eq. IO12 (cumulative plot only; - - - -) and M8 (

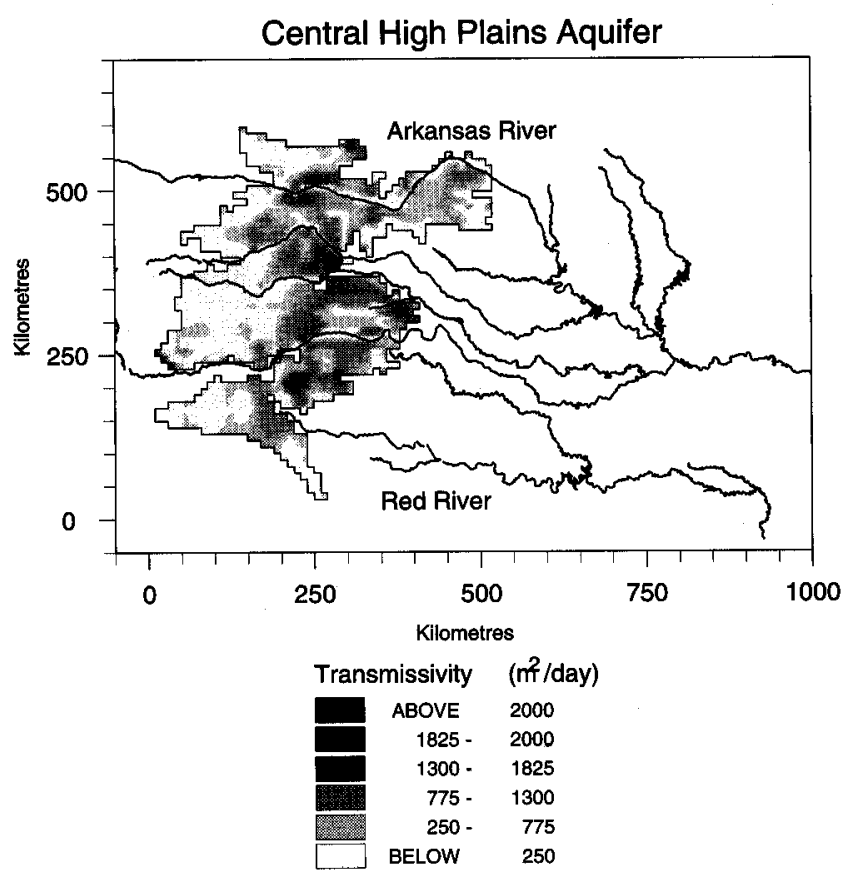

Fig. 10. Transmissivity map for the Central High Plains Aquifer, derived using $M 9$ 
most their addition would slightly alter the shapes and timing of the rising and falling limbs of the simulated river storm hydrographs. The methods in Ewen (1997) for modelling sub-grid routing of surface water have not been implemented. If implemented, the peak flows in the rivers would be slightly delayed and attenuated. The implementation could allow for surface flows taking place between gridsquares and for flooding of gridsquares by water from the main river network.

The input and output equations are, in effect, equations for large-scale hydrology. It is worth noting that, with the exception of the spatial integration of the snowmelt equation, the only aggregation process used in deriving the input and output equations and evaluating their parameters is the simple summation of fine-scale data.

Information on the sub-grid moisture status and spatial distribution is incorporated explicitly in the input and output equations. It is also, however, incorporated implicitly via the primary state variables, as can be seen by considering the role of $S_{g}$ in the term $\delta_{3} S^{s} S_{g}$ in the exfiltration equation, IO12. Putting to one side the complicating factor $S^{s}$, this term accounts for the sensitivity of large-scale exfiltration to large-scale storage, as is implicit in the aggregated M8 time-series, for exfiltration and storage, which were used to calibrate $\delta_{3}$. This sensitivity, however, simply accounts for an effect seen at the fine scale, where increasing wetness usually gives rise to increasing exfiltration.

Figure 8 shows the root zone storage. For every hour, the change in storage depends on the net input, which in turn depends on storage. The results in the figure show, however, that the system of input and output equations is robust to changes in storage, since the simulated storage does not drift unphysically high or low with time. Testing using split-sample verification showed that they are also robust to changes in the forcing variables.

It may be argued that the input and output equations are not parsimonious in parameters; but it is probably premature in the development of the UP approach to get overly concerned with parsimony.

The parameter sets in Table 6 for Little Washita and McAlester were both derived using the fine-scale basin physical data for Little Washita, and are different only as a result of the precipitation and meteorological data used. It may be that other input and output equations can be found which show less parameter sensitivity to the precipitation and meteorological data, or even no significant sensitivity. Whether such equations are 'better' (e.g. in the sense of being more generally applicable) than the equations presented here would require investigation.

The work on the ARRB model is not yet complete, and much remains to be done, especially to investigate sensitivities to changes in land-use and climate. However, the work presented here and the ARRB simulation work described in Kilsby et al. (1999) give a clear, positive, indication of the nature and quality of what is currently practical using large-scale physically-based hydrological modelling.

\section{Acknowledgements}

Claire Fallows ran most of the M7 simulations and helped collate the data for the parametrization basins, and Steve Birkinshaw and Greg O'Donnell helped in the development and testing of model M7. This work was funded by the UK Natural Environment Research Council (NERC), under Grant GST/02/629, as part of the Terrestrial Initiative in Global Environment Research (TIGER), and was carried out using a UNIX workstation funded jointly by United Kingdom Nirex Limited and NERC grant GR3/E0009.

\section{References}

Ewen, J., 1997. 'Blueprint' for the UP modelling system for large-scale hydrology, Hydrol. Earth System Sci., 1, 55-69.

Kilsby, C. G., Ewen, J., Sloan, W.T. and Fallows, C. S., 1999. The UP modelling system for large scale hydrology: simulation of the Arkansas-Red River basin, Hydrol. Earth System Sci., 3, 137-149.

Naden, P., Broadhurst, P., Tauvernon, N. and Walker, A., 1999. River routing for global climate models, Hydrol. Earth System Sci., 3, 109-124.

Press, W. H., Flannery, B. P., Teukolsky, S. A. and Vetterling, W. T., 1992. Numerical Recipes. Cambridge Univ. Press, Cambridge, UK. 